\title{
Spatial Distribution of Urban Transportation Capacity and Its Influencing Factors: A Case Study of Guangdong
}

\author{
Yannong Xu \\ Hong Kong and Macao Institute of Economics, Jinan University, Guangzhou, China \\ Email: ama_xu1@163.com
}

How to cite this paper: Xu, Y.N. (2019) Spatial Distribution of Urban Transportation Capacity and Its Influencing Factors: A Case Study of Guangdong. Modern Economy, 10, 334-346.

https://doi.org/10.4236/me.2019.101022

Received: December 28, 2018

Accepted: January 20, 2019

Published: January 23, 2019

Copyright (อ 2019 by author(s) and Scientific Research Publishing Inc. This work is licensed under the Creative Commons Attribution International License (CC BY 4.0).

http://creativecommons.org/licenses/by/4.0/

\section{(c) (i) Open Access}

\begin{abstract}
Using spatial autocorrelation analysis method, the spatial and temporal evolution of the overall development of Guangdong's transportation capacity during 2001-2016 is analyzed. There is no large displacement between high-level and low-level agglomeration areas between cities, and there is obvious path dependence. According to the development level of transportation capacity of 21 prefecture-level cities in 2010-2016, the factors affecting the development level of transportation capacity are discussed. The results show that: Guangdong's overall transportation capacity has increased with time, and transportation resources have been concentrated in Guangzhou in recent years. Cities in the high-level transportation capacity segment and cities in the low-level transportation capacity segment are relatively fixed, and there is path dependence; transportation fixed assets investment, transportation industry employees, and economic development level improve the transportation capacity. There is a significant positive impact; government fiscal expenditure and industrial structure have a significant negative impact on transportation development, reflecting the ineffectiveness and mismatch in the process of government fiscal expenditure and the unreasonable industrial structure; population density has no obvious development of transportation capacity. As the population density increases, traffic congestion in the city will not produce a good effect on the improvement of transportation capacity.
\end{abstract}

\section{Keywords}

Urban Transport Capacity, Spatial Distribution, Influencing Factors

\section{Introduction}

Transportation is the pioneering industry and basic industry of the national 
economy. It is also an important part of the productive service industry. It not only has its own direct economic contribution, but also can closely link economic activities in different regions and reduce transportation costs and transactions to a certain extent. Costs generate positive spatial spillovers in surrounding areas; increased transport capacity increases regional accessibility, attracts more elements to gather, and exerts agglomeration economic effects. According to the new economic geography, transaction costs are the resistance of economic activities. As a kind of transaction cost, transportation cost hinders the occurrence of new economic activities to a certain extent; the transaction subject and factor resources are in different spatial positions, and the resistance to the transaction is reached. The size will vary. The smoothness and high efficiency of transportation can weaken the spatial contradiction in economic transactions, promote the flow of factor space, reduce the transportation cost and improve the transportation capacity, which can better realize the location choice of enterprises and residents. The improvement of transportation capacity also means the economic activity has reduced resistance and the elements are better distributed in different spaces [1] [2] [3].

With the continuous deepening of economic development, the lag of transportation capacity will restrict economic development; in recent years; Guangdong's investment in transportation infrastructure has increased year by year. Under such investment, what is the actual situation of the transportation capacity of various cities in Guangdong Province? Does investment bring about a significant increase in transportation capacity, and what are the main factors affecting the development of transportation capacity? This paper takes the city as a spatial unit to study the spatial distribution of urban transport capacity in Guangdong, analyzes and evaluates the changes of transportation capacity of different cities in different time sections, helps to understand the spatial and temporal changes of Guangdong's urban transport capacity, and explores the transport capacity. The influencing factors and the use of panel data from prefecture-level cities for empirical research provide reasonable advice for improving regional transport capacity.

\section{Literature Review}

Due to the existence of transportation costs, the development of transportation has an important impact on economic activities. The research results on this issue are also abundant. Most scholars' research perspectives are based on the transportation infrastructure. Investment in facilities can reduce transportation costs and thus promote economic activity. The research direction is mainly to consider the effects of transportation infrastructure on economic growth and spillover effects. Hu Angang, Liu Shenglong [4] found that the economic growth rate of transportation investment was $1.38 \%$ by studying the traffic investment data from 1985 to 2006, and the contribution of spillover effect was about $0.29 \%$. Liu Shenglong and Hu Angang [5] studied the spillover effects of infrastructure 
on economic growth. Transportation infrastructure has a significant spillover effect on China's economic growth. For every $1 \%$ increase in transportation infrastructure, China's TFP increased by $0.378 \%$. Zhang Xueliang [6] believes that the fundamental reason for the spatial spillover effect of transportation infrastructure on economic growth lies in its regional externalities. Using spatial measurement methods to study the spatial spillover effects of China's transportation infrastructure on regional economic growth, it is found that the impact of other regional transportation bases on local economic growth is mainly positive spillover. Lin Xiongbin, Yang Jiawen [7] explored the relationship between provincial transportation investment, labor force and GDP in 1997-2013, and found that transportation investment played an obvious spillover effect in national economic growth, but the elasticity of transportation investment in the eastern and northeast regions Higher than the central and western regions; there is a large spatial difference.

Since the improvement of transportation capacity is directly related to the reduction of transportation cost, the increase of transportation capacity means the increase of transportation product supply and the improvement of efficiency, which can meet more transportation demand, reduce the resources of factor resources, the resistance of population flow, and reduce transportation. Cost, so it is necessary to study the development of transportation capacity.

In the choice of proxy variables for transportation development, there are roughly two types of paths: one is the unified caliber of the currency form, and the other is the physical form. Per capita road area using physical form, railway road network density, operating line network density, annual public transport passenger traffic, etc [8] [9]. The use of currency is mainly based on transportation investment, such as the use of transportation investment in 31 provinces across the country [7], and transportation investment using the Ministry of Communications' first-hand information [4]. However, due to the public goods attribute of transportation, the decision of investment amount does not completely follow the principle of maximizing economic benefits, and the investment price index is difficult to reflect the actual stock level; therefore, there is not necessarily a certain inevitability between transportation infrastructure investment and transportation capacity. The use of physical form indicators can better reflect the situation of transportation infrastructure [8]. This paper draws on the above scholars' viewpoints and uses the indicators of physical objects as a substitute for transportation capacity.

At present, there are not many literatures on the factors affecting transportation capacity; the more population density, the higher the level of urbanization, the degree of industrial structure and industrialization have an effect on transportation capacity by affecting transportation demand. Li Hairong [10] analyzes the mechanism of the factors affecting transportation facilities, mainly using population density and industrial production value. Chen Yinxi, Sun Qiong [9] analyzed the level of infrastructure by using a series of influencing factors such 
as private capital participation, local government fiscal expenditure level, economic development level, and control variables. Tan Jianxin, Yang Jinli [11] use population density, urbanization level, per capita income, industrial structure, inter-regional transportation demand level as demand factors; unit transportation cost of regional transportation infrastructure; financial strength of potential investors; Factors to study its impact on infrastructure.

\section{The Evolution of the Spatial Pattern of the Development of Transportation Capacity}

The annual freight volume and passenger traffic data of various cities in Guangdong are used for standardization processing, and the values after standardization processing are added as the transport capacity proxy variables. In order to make the transport capacity index non-negative, the standardization method selects the normalization method. The specific formula is: (X-Min)/(Max-Min) [12], in order to facilitate the observation of the distribution level of Guangdong's transport capacity development from the overall shape. Dynamic characteristics, select four time-section data in 2001, 2005, 2010 and 2016, and use ArcGIS10.0 software to classify the development level of transportation capacity into low level, medium level, medium level and medium height according to the natural fracture point clustering method. The level and level of the five levels are analyzed to analyze the spatial distribution of development levels.

As shown in Figure 1, The five colors range from dark to light and represent high levels, medium to high levels, medium to low levels, and low levels of transportation capacity. From the perspective of overall spatial distribution, the spatial distribution of transportation capacity is not balanced. The spatial distribution of transportation capacity from west to east alternates with high and low levels. There are few cities with high level of transportation development, and many cities with low and medium-low levels. Guangzhou has always been The city with the strongest transportation capacity; the transportation capacity of the surrounding cities surrounding Guangzhou is also relatively high, at a medium to high level. In the middle part of Guangdong from west to east, it is concentrated in a region with good transportation capacity. This area is in both directions. In order to transport low-level gathering areas, the phenomenon of low concentration to the east and west has improved.

From the specific changes, in addition to the small high-level areas formed in

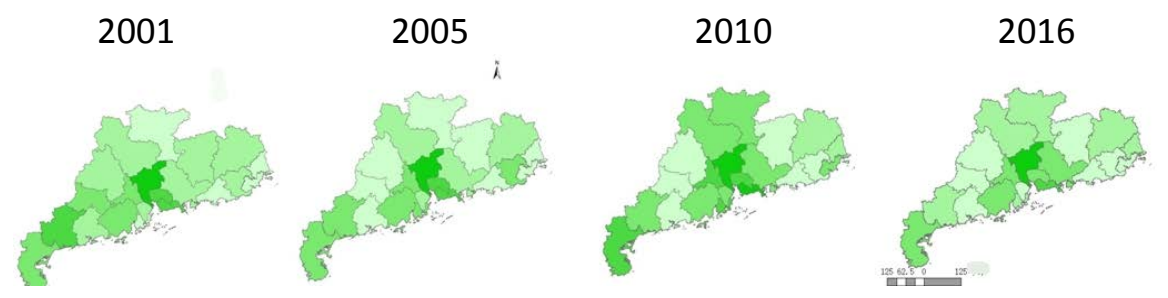

Figure 1. Grade map of urban transport capacity in Guangdong Province in 2001, 2005, 2010 and 2016. 
Guangzhou, Dongguan and Shenzhen in 2001, the gap in transportation capacity of most other cities is not significant, and the spatial distribution of the alternating areas from west to east has not yet formed. In 2005, the transportation capacity of Zhaoqing, Yunfu and Jiangmen declined to form a low-level area. The middle-class cities of Guangdong initially formed a triangular area with high-level and medium-high level of transportation centered on Guangzhou; Heyuan City fell into a low-level area of transportation development; The spatial distribution of high and low levels of transportation from west to east. In 2010, with the continuous improvement of the overall economic level of Guangdong, the demand for transportation capacity has also increased due to the pooling of population and resources. In the context of increasing investment in transportation, the overall transportation capacity of Guangdong has increased. The phenomenon of unbalanced transportation space has further intensified, and the spatial pattern of alternating high and low is remarkable. In 2016, the spatial distribution pattern of transportation capacity has not changed much, but the overall transportation capacity has declined, especially in the surrounding cities centered on Guangzhou. From 2001 to 2016, the transportation capacity of major cities has increased and declined, but there has not been a large displacement in the high-level and low-level agglomeration areas, and there is obvious path dependence.

\section{The Establishment of the Model}

\subsection{Selection of Variables}

Explain the variable transportation capacity. New economic geography views transportation costs as an endogenous variable of economic growth [13]. Good transportation capacity helps to reduce transaction costs and thus promote economic growth. This paper selects the standardized data of annual freight volume and passenger traffic index of each city in Guangdong as an indicator to reflect the transportation capacity.

Explain the selection of variables. 1) Fixed assets investment. Transportation investment can improve the level of transportation infrastructure, reduce transportation costs, improve transportation capacity, and promote economic growth [7]. This paper uses the proportion of fixed assets investment stations in the city's transportation industry as a substitute variable.

2) The level of fiscal expenditure. Most of the local government's fiscal expenditures are used for the construction of various general public goods. The increase in fiscal expenditures is conducive to the provision of public transportation products, thereby improving transportation capacity [14] [15]. This paper uses the financial expenditure level (100 million yuan) of local governments in each city as data support.

3) Practitioners. This article reflects the number of employees in the city's transportation industry at the end of the year.

Control variable selection. 1) Population density. The density of urban popu- 
lation also has an impact on transportation capacity. Cities with too small population density have little ability to gather resources, transportation is not developed, and transportation capacity is low. The increase in population density has increased the traffic burden and has a negative impact on transport capacity. In this paper, the ratio of the population of the city to the administrative area of the city as the indicator of urban population density is 10,000 people per square kilometer.

2) Industrial structure. According to the new economic geography, in addition to pursuing the advantages of factor endowment, reducing transaction costs is the decisive factor for the industry to start gathering in one place; the concentration of industry will change the industrial structure of a place to promote the upgrading of industrial structure. Different industries have different requirements for transportation volume, and changes in industrial structure can change transportation demand and have an effect on transportation capacity. Therefore, the ratio of the tertiary industry to the secondary industry in each city is selected as a variable to measure the degree of urban industrial structure.

3) The level of economic development. A good economic foundation is a precondition for the investment expenditure to play a role. The region with a high level of economic development has a strong ability to gather resources, and the role of transportation investment in improving transportation capacity is more obvious. This paper takes GDP (GDP) of each city as one of the control variables affecting urban transport capacity.

\subsection{Data Description}

The explanatory variable transport capacity index uses the annual freight volume and passenger traffic data of each city in Guangdong. The data of these two parts are different, so the standardization process is performed, and the values after the standardization process are added as the transport capacity proxy variables. In order to make the transport capacity index non-negative, the standardization method selects the normalization method. The specific formula is: (X-Min)/(Max-Min), and the freight volume is added to the value after standardization of passenger traffic. The transport capacity proxy variable varies between 0 and 2. Table 1 below gives the values of the interpreted variables in the regression analysis section.

As shown in Table 1, the overall transportation capacity of Guangdong has increased with time. The cities that have maintained good growth momentum are mainly Guangzhou, Shaoguan, Yangjiang, Zhanjiang, Maoming and other cities. At the same time, some cities have gradually reduced their transportation capacity. These cities mainly include Zhuhai and Dongguan. Shenzhen, Qingyuan, Jieyang. From the perspective of transportation capacity, Guangzhou, Shenzhen, Shaoguan and Dongguan are the transportation hub cities in the initial years, but only Guangzhou has maintained a good growth trend and maintained its position as the first city in transportation in the past three years, despite Shenzhen, The transportation development in Shaoguan City is better than 
Table 1. Evaluation of transportation capacity of cities in Guangdong.

\begin{tabular}{|c|c|c|c|c|c|c|c|}
\hline & 2010 & 2011 & 2012 & 2013 & 2014 & 2015 & 2016 \\
\hline Guangzhou & 1.302 & 1.307 & 1.302 & 1.333 & 2.000 & 2.000 & 2.000 \\
\hline Shenzhen & 1.472 & 1.488 & 1.488 & 1.401 & 0.346 & 0.378 & 0.394 \\
\hline Zhuhai & 0.239 & 0.230 & 0.220 & 0.220 & 0.114 & 0.129 & 0.132 \\
\hline Shantou & 0.048 & 0.040 & 0.037 & 0.035 & 0.043 & 0.049 & 0.048 \\
\hline Foshan & 0.658 & 0.667 & 0.794 & 0.768 & 0.454 & 0.501 & 0.479 \\
\hline Shaoguan & 0.155 & 0.157 & 0.156 & 0.189 & 0.171 & 0.219 & 0.231 \\
\hline Heyuan & 0.036 & 0.028 & 0.027 & 0.030 & 0.046 & 0.060 & 0.067 \\
\hline Meizhou & 0.075 & 0.073 & 0.072 & 0.072 & 0.063 & 0.073 & 0.077 \\
\hline Huizhou & 0.241 & 0.269 & 0.294 & 0.304 & 0.239 & 0.285 & 0.294 \\
\hline Shanwei & 0.024 & 0.035 & 0.042 & 0.048 & 0.000 & 0.000 & 0.000 \\
\hline Dongguan & 0.688 & 0.666 & 0.639 & 0.576 & 0.200 & 0.204 & 0.192 \\
\hline Zhongshan & 0.216 & 0.206 & 0.293 & 0.337 & 0.170 & 0.201 & 0.175 \\
\hline Jiangmen & 0.245 & 0.232 & 0.221 & 0.202 & 0.228 & 0.239 & 0.248 \\
\hline Yangjiang & 0.027 & 0.024 & 0.035 & 0.042 & 0.061 & 0.095 & 0.101 \\
\hline Zhanjiang & 0.179 & 0.183 & 0.201 & 0.182 & 0.187 & 0.219 & 0.245 \\
\hline Maoming & 0.116 & 0.119 & 0.123 & 0.120 & 0.147 & 0.159 & 0.169 \\
\hline Zhaoqing & 0.071 & 0.062 & 0.060 & 0.053 & 0.060 & 0.070 & 0.074 \\
\hline Qingyuan & 0.169 & 0.171 & 0.169 & 0.166 & 0.123 & 0.151 & 0.161 \\
\hline Chaozhou & 0.023 & 0.022 & 0.025 & 0.027 & 0.027 & 0.033 & 0.038 \\
\hline Jieyang & 0.035 & 0.033 & 0.029 & 0.026 & 0.022 & 0.023 & 0.024 \\
\hline Yunfu & 0.046 & 0.040 & 0.037 & 0.038 & 0.039 & 0.053 & 0.062 \\
\hline
\end{tabular}

that in Guangzhou, but the development trend is reversed, especially in recent years, which is in stark contrast with Guangzhou's transportation capacity. This also reflects the phenomenon that transportation resources are concentrated in Guangzhou; in addition, Shantou The development level of Meizhou's transportation has not changed much, but it has been at a low level. From the perspective of the trend of transportation capacity, Foshan, Huizhou, Shanwei and Zhongshan show a change of first increase and then decrease, while Heyuan, Jiangmen, Zhaoqing and Yunfu are opposite. Cities with high levels of transport capacity and cities with low levels of transport capacity are relatively fixed and path dependent.

In addition, the original data of industrial structure, economic development level, fixed asset investment, and fiscal expenditure level are derived from the Guangdong Statistical Yearbook 2011-2017; the population density, employees and transportation capacity indicators are derived from the 2011-2017 China Urban Statistics Yearbook. Finally, the cross-sections of 21 prefecture-level cities in Guangdong, 147 observations, were used to estimate the model using Stata12.0. 
In order to further explore the factors affecting transportation capacity, combined with the calculation and analysis of transportation capacity indicators and the empirical research of existing scholars, the following measurement models are established:

$$
\text { Trans }_{i t}=\alpha+\beta \text { inves }_{i t}+\gamma \text { govex }_{i t}+\delta \text { emplo }_{i t}+\theta X_{i t}+\varepsilon_{i t}
$$

where: $i$ represents the city; $t$ represents the time; the explanatory variable trans is the transportation capacity level; the explanatory variables are the inves of the fixed assets investment of the municipal transportation industry, the fiscal expenditure level of the local governments, the govex, and the employees of the municipal transportation industry; $X$ represents the set of control variables of the model, involving population density (popd), industrial structure (struc), economic development level (gdp); $\alpha, \beta, \gamma, \delta, \theta$ are parameters to be estimated; $\varepsilon$ is a random error term.

\section{Estimation Results and Analysis}

\subsection{Inspection of Model Selection}

The panel data used in this paper is the balance panel data. The analysis of the panel data model mainly includes the hybrid estimation model, the fixed effect model and the random effect model. If the slope and intercept do not differ significantly between the time series and the different individuals, then the model estimate can be mixed, i.e., the parameter estimation can be performed using the ordinary least squares method. If the slopes of the models are the same for different individuals or different time series, but the intercepts are different, it is necessary to check whether the intercept term is a fixed parameter or the average effect of the cross-section random error term and the time random error term; the former uses fixed The effect model, the latter can use the random effects model. In the method selection of the panel data model form, the F-test is generally used to determine whether to use the hybrid model or the fixed-effect mod$\mathrm{el}$, and then the Hausen method is used to test the fixed effect and the random effect model. According to the panel data and model assumptions of this paper, the results of $\mathrm{F}$ test using Stata12.0 statistical software are as follows: $\mathrm{F}=10.36$, p-value $=0.000$, the results show that the original hypothesis is rejected, that is, the slope and intercept of the model are not changed. The mixed OLS estimation method is discarded; then the Hausman test is used to use the fixed effect or the random effect model.

As shown in Table 2, the result is: $\operatorname{chi} 2(4)=46.67$, p-value $=0.000$. The results show that the alternative effect model is selected by accepting the alternative hypothesis.

\subsection{Model Estimation Results}

As shown in Table 3, from the perspective of the estimated coefficient of explanatory variables, the estimated coefficient of local government fiscal expenditure variable is -0.0008 , indicating that the increase of local government expenditure 
Table 2. Hausman test results.

\begin{tabular}{ccccc}
\hline & $(\mathrm{b})$ & $(\mathrm{B})$ & $(\mathrm{b}-\mathrm{B})$ & $\mathrm{sqrt}\left(\operatorname{diag}\left(\mathrm{V} \_\mathrm{b}-\mathrm{V} \_\mathrm{B}\right)\right)$ \\
& $\mathrm{fe}$ & $\mathrm{re}$ & Difference & S.E. \\
\hline gdp & 0.0000 & 0.0001 & -0.0001 & 0.0000 \\
struc & 0.0448 & -0.0746 & 0.1193 & 0.1748 \\
popd & -22.7226 & -0.4502 & -22.2724 & 4.4592 \\
inves & -0.7993 & 0.9492 & -1.7485 & 0.3686 \\
emplo & 0.0496 & 0.0362 & 0.0133 & 0.0197 \\
govex & -0.0005 & -0.0008 & 0.0002 & 0.0004 \\
\hline
\end{tabular}

Test: Ho: difference in coefficients not systematic. $\operatorname{chi} 2(7)=(\mathrm{b}-\mathrm{B})^{\prime}\left[\left(\mathrm{V} \_\mathrm{b}-\mathrm{V} \_\mathrm{B}\right)^{\wedge}(-1)\right](\mathrm{b}-\mathrm{B})=46.67$ Prob $>$ chi $2=0.000 . \mathrm{b}=$ consistent under Ho and Ha; obtained from xtreg; $\mathrm{B}=$ inconsistent under Ha, efficient under Ho; obtained from xtreg.

Table 3. Data estimation results.

\begin{tabular}{ccccc}
\hline \multirow{2}{*}{ Interpret variable } & \multicolumn{3}{c}{ Interpret variable trans } \\
\cline { 2 - 5 } & Expected symbol & Coefficient & S.D & Z value \\
\hline C & & 0.0592 & 0.0979 & 0.60 \\
gdp & + & $0.0001^{* * *}$ & 0.0000 & 6.90 \\
struc & + & $-0.7456^{* *}$ & 0.1066 & -0.70 \\
popd & + & -0.4502 & 0.3711 & -1.21 \\
inves & + & $0.9492^{* *}$ & 0.3847 & 2.47 \\
emplo & + & $0.0362^{* * *}$ & 0.0081 & 4.48 \\
govex & + & $-0.0008^{* * *}$ & 0.0002 & -4.62 \\
\hline
\end{tabular}

$\mathrm{R}$-sq $=0.8172, \mathrm{Obs}=126, \mathrm{Prob}>\operatorname{chi} 2=0, \mathrm{Chi} 2(6)=420.4$. Note: The test statistic is in parentheses, ${ }^{* *}$ and ** represent the $1 \%$ and $5 \%$ significance levels, respectively.

by 100 million will result in a decrease of transport capacity of 0.0008 , and local government fiscal expenditure will not improve transportation capacity, which is expected. The opposite is true; rapid urbanization has made the demand for transportation products in the city increase drastically. The construction funds for public goods in transportation are mainly government fiscal expenditures, but the mismatching and invalidation of resources generated in the process of government fiscal expenditure is difficult for transportation. The provision of public goods has a beneficial effect, thereby inhibiting the improvement of transportation capacity. On the other hand, the coefficient of fixed assets investment in transportation is positive, and it has passed the test at the level of significance of $1 \%$, indicating that the proportion of fixed assets investment in fixed assets investment in the whole society has increased by 1 , and the transportation capacity has increased by 0.9492 . Guangdong Province is located on the coast, with good social and economic development, concentrated population, easy to play agglomeration effect, and higher average income from transportation fixed assets investment, which has played a guiding role in improving transportation capacity. The transportation industry employees are significantly 
and positively related to the transportation capacity. For every 10,000 employees, the transportation capacity will increase by 0.0362 . The investment in the transportation industry will have a higher contribution to the improvement of transportation capacity.

From the coefficient of the control variable, the economic development level variable gdp is significantly positively correlated with the transportation capacity, the estimated coefficient is 0.0001 , the transportation capacity will increase by 0.0001 for every 100 million yuan increase in gdp, and the transportation product in the region with good economic development level. The demand is large, and the construction of transportation infrastructure is also increasing, which is conducive to the promotion of transportation capacity. The coefficient of the population density variable is negative, contrary to the expected sign and the coefficient has not passed the test; in general, the population density is more likely to agglomerate resources, the transportation demand is huge, and the transportation capacity is improved; but with the continuous scale of the city Expanded, many cities with better coastal economic levels have big city diseases, and the population is too dense, leading to traffic congestion, but it has a restraining effect on the improvement of transportation capacity. The significant negative correlation coefficient between industrial structure and transportation capacity is -0.7456 . The $5 \%$ significance level test indicates that the current freight demand brought by the secondary industry has increased more than that brought by the tertiary industry such as tourism and modern logistics. The total demand has increased; indicating that the upgrading of industrial structure does not promote the development of transportation. This may be because the industrial structure is not reasonable enough, and the lack of industrial structure is not good enough to reduce the transportation cost. Therefore, the transportation capacity Raising will not help.

\section{Main Conclusions and Recommendations}

Based on the spatial autocorrelation analysis method and the total passenger and freight traffic data, the spatial and temporal evolution of the overall development of Guangdong's transportation capacity during 2001-2016 was analyzed. Based on this, the fixed panel model was used to explore the 21 prefectures affecting Guangdong in 2010-2016. The factors of the development level of transportation capacity of prefecture-level cities show that:

1) In the overall space of Guangdong, the overall transportation capacity has increased with time. From 2001 to 2014, the transportation capacity of major cities has increased and declined. The city that has maintained good growth momentum is mainly Guangzhou and has been in the past three years. Maintaining the status of the first city in transportation, although some cities have just begun to develop better than Guangzhou in terms of transportation, the development trend is reversed, especially in recent years, in stark contrast to Guangzhou's transportation capacity, which also reflects the concentration of transportation 
resources in Guangzhou. The phenomenon, but the high-level agglomeration area is centered on Guangzhou, and the low-level agglomeration area is in the northeastern city. There is no large displacement between the high-level and low-level agglomeration areas, and there is obvious path dependence.

2) The three factors of transportation fixed assets investment, transportation industry employees and economic development level have a significant positive impact on the improvement of transportation capacity. Guangdong Province is located in the coastal areas, with good social and economic development and good economic development. The demand for transportation products is large, and the construction of transportation infrastructure is also increasing, which is conducive to the promotion of transportation capacity. At the same time, the population of Guangdong is concentrated, and it is easy to play the agglomeration effect. The average income of transportation fixed assets investment is higher. It has played a guiding role in improving transportation capacity; for every 10,000 employees, the transportation capacity will increase by 0.0362 ; the investment in the transportation industry will have a higher contribution to the improvement of transportation capacity.

3) The government's fiscal expenditure and industrial structure have a significant negative impact on the development of transportation. It reflects the ineffectiveness and mismatch in the process of government fiscal expenditure, the unreasonable industrial structure, and the lack of industrial structure. The population density has no development in transportation capacity. The obvious effect is mainly because with the increasing population density, many cities with better coastal economic levels have traffic congestion, but they have no good effect on the improvement of transportation capacity. Based on this, the paper proposes the following suggestions:

First, further increase the proportion of transportation fixed assets investment, strive to improve the quality of transportation infrastructure construction; reduce the redundant construction of transportation facilities, strengthen the maintenance of existing facilities, and improve their utilization efficiency.

Second, improve the efficiency of government fiscal expenditure. The fixed assets investment in the transportation industry has a positive effect on the improvement of transportation capacity. However, the government's fiscal expenditure cannot improve the transportation capacity. Government expenditure should be more targeted, and the direction and quantity of expenditure should be correctly chosen to avoid duplication and ineffective construction.

Third, increase the number of employees in the transportation industry. As a productive service industry, the transportation industry is responsible for absorbing a large number of labor and employment. Increasing the number of employees in the transportation industry can create more jobs. At the same time, improving transportation capacity and improving urban traffic congestion from improving transportation management planning, Improve the efficiency of the entire transportation network.

Fourth, vigorously develop the tertiary industry. During the period of devel- 
opment of the secondary industry, the total industrial value increased rapidly and the transportation demand increased greatly. When the industry develops to a certain extent, the tertiary industry is valued, the frequency of international trade increases, and the products are rapidly circulated. Together with the development of tourism and modern logistics, the total demand for transportation is still rising. In general, the upgrading of industrial structure can promote the development of transportation, and it is a long-term mechanism to continuously improve the transportation capacity from the demand side.

Fifth, rationally control the size of the city and prevent diseases in big cities. Population agglomeration increases the demand for transportation products in cities, and promotes the increase of transportation supply, thereby promoting the improvement of transportation capacity. At present, the population density of some cities is too large, and the growth of transportation capacity cannot cope well with the rapidly expanding urban population. The scale of the city cannot be expanded without limit, and the management efficiency of transportation is improved.

\section{Conflicts of Interest}

The author declares no conflicts of interest regarding the publication of this paper.

\section{References}

[1] Han, W. and Zhang, Z.M. (2015) Transportation and China's Economic Growth-Based on the Perspective of "Transportation Costs". Exploring Economic Issues, No. 8, 14-21.

[2] Feng, W. and Xu, K.N. (2013) Transportation Infrastructure and Economic Growth: A Literature Review. Sankei Review, No. 3, 63-70.

[3] Li, P.X. and Liu, D.S. (1995) A Comparative Analysis of China's Infrastructure Level and Economic Growth. Management World, No. 2, 106-111.

[4] Hu, A.G. and Liu, S.L. (2009) Transportation, Economic Growth and Spillover Effects: Based on the Results of China's Inter-Provincial Data Space Economic Measurement. China Industrial Economy, No. 5, 5-14.

[5] Liu, S.L. and Hu, A.G. (2010) Inspection of the Externalities of Infrastructure in China: 1998-2007. Economic Research, No. 3, 4-15.

[6] Zhang, X.L. (2012) Does China's Transportation Infrastructure Promote Regional Economic Growth? Concurrently on the Spatial Spillover Effect of Transportation Infrastructure. Chinese Social Sciences, No. 3, 60-77+206.

[7] Lin, X.B. and Yang, J.W. (2016) Time and Space Evolution of China's Transportation Investment and Its Economic Spillover Effect-The Empirical Demonstration of Provincial Panels from 1997 to 2013. Geography Research, No. 9, 1727-1739.

[8] Liu, B.L., Wu, P. and Liu, Y.H. (2010) Transportation Infrastructure and China's Total Factor Productivity Growth: Spatial Panel Measurement Analysis Based on Provincial Data. China Industrial Economy, No. 3, 54-64.

[9] Chen, Y.X. and Sun, Q. (2016) Estimation and Influencing Factors of China's Infrastructure Development Level-An Empirical Study Based on Provincial Panel Data. 
Economic Geography, No. 8, 23-30.

[10] Li, H.R. (2014) Research on Spatial Structure of Resource-Based Urban Infrastructure Based on GIS-Taking Panzhihua City as an Example. Chengdu University of Technology, Chengdu.

[11] Tan, J.X. and Yang, J.L. (2009) Spatial Distribution of Transportation Infrastructure and Regional Economic Growth. Journal of Yunnan University for Nationalities (Philosophy and Social Sciences Edition), No. 7, 101-105.

[12] Yang, Y. (2007) Analysis of Weighting Methods in Multi-Index Comprehensive Evaluation. Statistics and Decision, No. 7, 17-19.

[13] Wang, S.B. and Guo, J.K. (2017) Time-Space Evolution and Spatial Correlation Analysis of the Development of Urban Overall Transportation Flow in China. Arid Zone Resources and Environment, No. 2, 43-49.

[14] Zhao, W. (2012) Study on the Temporal and Spatial Pattern Evolution of County Economic Development in Shanxi Province. Shaanxi Normal University, Xi'an.

[15] Li, Z.M., Liu, Y.H. and Zhang, Q. (2011) An Empirical Study of the Economic Growth of the "New Silk Road" Transportation Economic Belt-A Panel Data Model Based on Six Factors Such as Human Capital. Economic Research, No. 1, 77-80. 